\title{
Minimally invasive transforaminal lumbar interbody fusion with percutaneous navigated guidewireless lumbosacral pedicle screw fixation
}

\author{
Kevin S. Chen, MD, and Paul Park, MD \\ Department of Neurosurgery, University of Michigan, Ann Arbor, Michigan
}

This video details the minimally invasive approach for treatment of a symptomatic Grade II lytic spondylolisthesis with high-grade foraminal stenosis. In this procedure, the use of a navigated, guidewireless technique for percutaneous pedicle screw placement at the lumbosacral junction is highlighted following initial decompression and transforaminal interbody fusion. Key steps of the procedure are delineated that include positioning, exposure, technique for interbody fusion, intraoperative image acquisition, and use of a concise 2-step process for navigated screw placement without using guidewires.

The video can be found here: https://youtu.be/2u6H4Pc_8To.

KEYWORDS minimally invasive; TLIF; percutaneous pedicle screw; image guidance; spinal fusion; spondylolisthesis; video 